
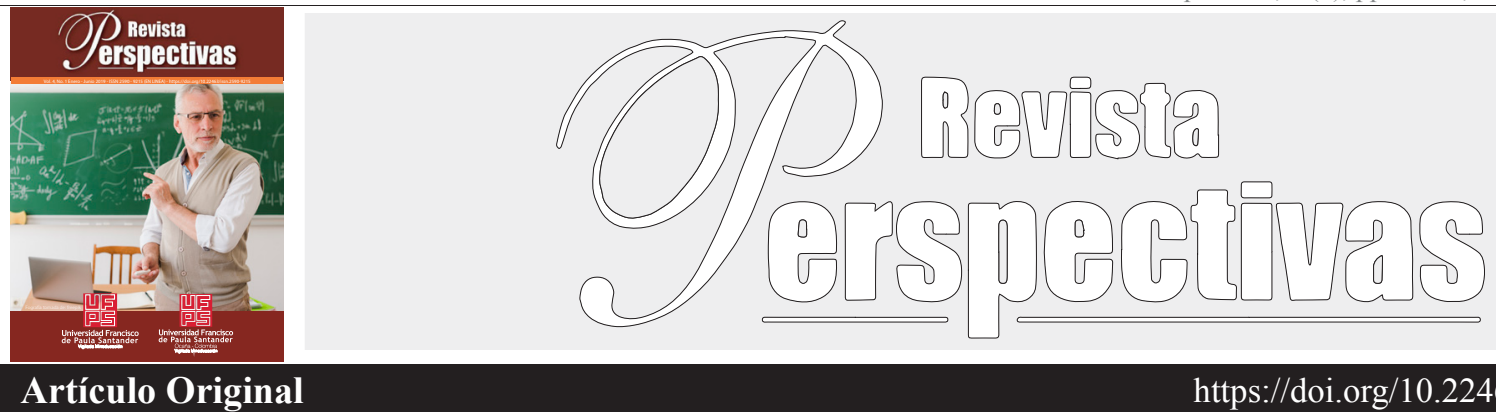

Artículo Original

https://doi.org/10.22463/25909215.1759

\title{
Estrategias para la enseñanza de la matemática: una mirada desde los docentes en formación
}

Strategies for teaching mathematics: a view from teachers in training

Estratégias para o ensino da matemática: uma visão dos professores em formação

Walter Byron Pineda-Izasa ${ }^{\mathrm{a}}$, César Augusto Hernández-Suárez ${ }^{\text {b* }}$, Olga Lucy Rincón-Leal ${ }^{\mathrm{c}}$

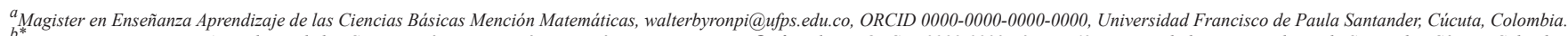

b* Magister en Enseñanza Aprendizaje de las Ciencias Básicas Mención Matemáticas, cesaraugusto@ufps.edu.co, ORCID 0000-0002-7974-5560, Universidad Francisco de Paula Santander, Cúcuta, Colombia.

${ }^{c}$ Magister en Matemáticas mención Educación, olgarincon@ufps.edu.co, ORCID 0000-0002-8080-496X, Universidad Francisco de Paula Santander, Cúcuta, Colombia.

Cómo citar: Pineda, W. B., Hernández, C. A. \& Rincón, O. L. (2019). Estrategias para la enseñanza de la matemática: una mirada desde los docentes en formación. Perspectivas, 4(1), 48-53.

Recibido: Agosto 20, 2018; Aceptado: Noviembre 01, 2018

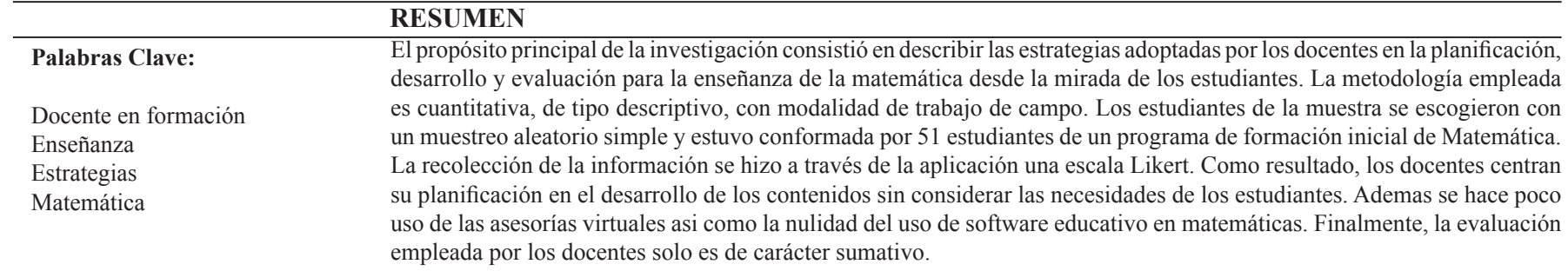

\section{ABSTRACT}

Keywords:

The main purpose of the research was to describe the strategies adopted by teachers in planning, developing and evaluating mathematics teaching from the students' point of view. The methodology used is quantitative, descriptive, and fieldwork. The students in the samplewere chosen withasimplerandomsampleand consisted of 51 students fromaninitialmathematics training program. The information was collected through the application of a Likert scale. As a result, teachers focus their planning on content development without considering the needs of the students. In addition, little use is made of virtual consultancies as

Education well as the nullity of the use of educational software in mathematics. Finally, the evaluation used by teachers is only summative.

Strategies

Mathematics

\section{RESUMO}

Palavras chave:

O objetivo principal da pesquisa foi descrever as estratégias adotadas pelos professores no planejamento, desenvolvimento e avaliação do ensino de matemática do ponto de vista dos alunos. A metodologia utilizada é quantitativa, descritiva e

Professor em formação

Formação Acadêmica de trabalho de campo. Os alunos da amostra foram escolhidos com uma amostra aleatória simples e composta por 51 alunos de um programa de treinamento inicial de matemática. A informação foi recolhida através da aplicação de uma escala de Likert. Como resultado, os professores concentram o seu planeamento no desenvolvimento de conteúdos sem ter em conta as necessidades dos alunos. Além disso, pouco uso é feito de consultorias virtuais, bem como a nulidade do uso de software educacional em matemática. Finalmente, a avaliação utilizada pelos professores é apenas sumativa.

\section{Introducción}

La búsqueda de la enseñanza de las matemáticas ha sido desde siempre una preocupación pedagógica (Jiménez, 2007). En primer término, porque ha aumentado exponencialmente el número de escuelas y otros centros de formación al reconocerse la educación como parte de los derechos fundamentales de

*Autor de Correspondencia.

E-mail: cesaraugusto@ufps.edu.co (César Augusto Hernández Suárez)

(C) $\Theta$ Peer review is the responsibility of the Universidad Francisco de Paula Santander.

This is an article under the license CC BY-ND las personas (Marchesi, Tedesco, \& Coll, 2009). Por otra parte, ha habido una importante revolución dentro de las ciencias de la educación, que han propuesto y probado numerosos métodos y estrategias para a enseñanza de las distintas materias (incluidas las matemáticas) objeto de estudio en las escuelas (Blanco, 2011).

Desde otra perspectiva, se distinguen, por una parte, el conocimiento de las estrategias de enseñanza de la matemática 
que los docentes conocen y por otra, las que realmente emplean. Una praxis limitada, en efecto, no proviene necesariamente del desconocimiento de las posibilidades y especificidades de la didáctica especial, sino que también puede deberse a que el docente, aun conociendo vías alternativas y mejor ajustadas a los propósitos de la enseñanza de la matemática, por rutina, falta de recursos o iniciativa termina por limitarse a un repertorio escaso e ineficiente de posibilidades.

De modo general se entiende que las estrategias didácticas se refieren a los procesos afectivos, cognitivos y procedimentales que facultan al docente llevar a cabo la instrucción y al estudiante construir el aprendizaje. En consecuencia, "las estrategias didácticas son fundamentalmente procedimientos deliberados por el ente de enseñanza o aprendizaje que poseen una intencionalidad y motivaciones definidas" (Feo, 2010, p. 221)

En ese sentido un profesor debe poseer conocimiento no solo de la disciplina que enseña, sino además el modo en que debe ser enseñada, es decir, de cuáles son las vías que la pedagogía ha probado como más apropiadas para alcanzar el dominio de los saberes disciplinares específicos por parte de los estudiantes. De esta simbiosis entre conocimiento disciplinar y pedagógico ha surgido como lugar de encuentro la didáctica especial, propia de cada campo de conocimiento.

Por otra parte, se insiste en que los estudiantes deben adquirir diversos tipos de conocimientos y desarrollar procesos matemáticos aplicables a situaciones diversas, lo cual supone profundizar sobre los correspondientes métodos de aprendizaje y, muy particularmente, sobre técnicas adecuadas para el desarrollo de la enseñanza.

En el caso de la enseñanza de la matemática, los últimos años ha sido un tiempo de cambios; los avances han tenido lugar principalmente en el ámbito teórico, pero su repercusión en las aulas no ha ido al mismo ritmo. La causa de este desfase al parecer obedece tanto a la escasa comunicación entre los docentes y los expertos en educación matemática como al hecho de que los docentes durante su formación y actualización no disponen todavía de información $\mathrm{y} / \mathrm{o}$ formación suficiente sobre estrategias didácticas para el desarrollo apropiado del proceso de aprendizaje y enseñanza de las matemáticas escolares (Mora, 2003).

Entre las estrategias propuestas para la enseñanza de la matemática se encuentran, por ejemplo, la resolución de problemas (Sánchez \& Fernández , 2003; Do Ponte, Brunheira, Abrantes, \& Bastos, 1998), El uso de los juegos como recurso didáctico para la enseñanza y el aprendizaje de las Matemáticas: estudio de una experiencia innovadora (Muñiz-Rodríguez, Alonso \& Rodríguez-Muñiz, 2014), la demostración (Serres, 2002), las aplicaciones y su proceso de modelación (Blum, 1985) entre otros. Como es natural, todas las estrategias mencionadas se fundamenten en las ciencias de la educación como a la matemática propiamente dicha (Mora, 2003)
En cuanto a los participantes, se ha decidido tener en cuenta la visión de los estudiantes acerca de la planificación, ejecución y evaluación de las estrategias. Este trabajo se refiere a la indagación sobre estrategias de enseñanza, que tienen que ver con la didáctica especial del saber disciplinar, empleadas por un grupo de docentes universitarios en la enseñanza de la matemática.

De acuerdo a lo expuesto, se considera que es necesario tener en cuenta el punto de vista de los estudiantes acerca de las estrategias empleadas en clase desde su perspectiva ya que en ocasiones algunos docentes tienden a emplearlas sin tener en cuenta las dificultades, necesidades o dudas que puedan tener ellos frente a estas y, más aún, cuando se trata de la matemática.

Es necesaria una mirada crítica de los estudiantes y particularmente el que se refiere a sus relaciones con los profesores durante la práctica pedagógica, como uno de los factores que condiciona e influye sus percepciones respecto a su propio aprendizaje (Covarrubias \& Piña, 2004) es decir, qué es lo que piensan de las estrategias de enseñanza de las matemáticas empleadas por sus docentes.

En tal sentido, esta investigación pretendió estudiar la participación de los estudiantes en el aula desde su perspectiva. Los estudiantes observan y consideran la enseñanza real, pero quien reflexiona sobre los procesos y acontecimientos de la clase y toma las iniciativas es el docente; y quienes actúan mecánicamente para ejecutar lo pensado y decidido por el maestro son los estudiantes. En las muy pocas ocasiones en las que éstos reflexionan para decidir se trata de asuntos banales, porque las cuestiones esenciales se las ha reservado el docente consciente o inconscientemente (Bretones, 1996 ).

Es por ello que se describió las estrategias adoptadas en la planificación, desarrollo y evaluación para la enseñanza de la matemática de un grupo de profesores de un programa de formación inicial de docentes en matemáticas de una universidad publica colombiana, desde la perspectiva de los estudiantes mediante un análisis cuantitativo, de carácter descriptivo, que garantizó una selección de los participantes con un sesgo definido: que estrategias emplean sus docentes.

\section{Materiales y métodos}

El método de investigación empleado en el presente estudio correspondió al cuantitativo. El nivel que se estableció fue de carácter descriptivo y de campo, a partir del autoreporte de los estudiantes matriculados en las asignaturas del eje de formación disciplinar de un programa de formación inicial de docentes en matemáticas, sobre cuáles son las estrategias, adoptadas en el proceso de enseñanza, que conocen y usan los docentes titulares de estas asignaturas. 
Población y muestra. La Universidad Francisco de Paula Santander, en Cúcuta - Colombia, es una Institución de Educación Superior publica. Para el II semestre de 2017, la Licenciatura en Matemática, programa de formación inicial de docentes de la Facultad de Educación, Artes y Humanidades, tiene 133 estudiantes matriculados, distribuidos en diez semestres académicos. La muestra se escogió mediante un muestreo aleatorio simple, con un nivel de confianza del $92 \%$ y margen de error del 7,8 \% (Pardo \& Ruíz, 2002), lo que determinó un tamaño muestral de 51 estudiantes.

Instrumento. Como herramienta de recolección de la información se diseño y utilizo instrumento para los estudiantes que contenía 33 ítemes, de los cuales tres (3) fueron de carácter sociodemográfico y 30 estaban organizados en tres (3) escalas Likert, cada una con un conjunto de indicadores para medir la frecuencia con la que los docentes emplean las estrategias teniendo en cuenta la planificación, el desarrollo y la evaluación en el proceso de enseñanza de la matemática. Estas escalas tenían cinco opciones de respuesta: Nunca (1), Rara vez (2), Algunas veces (3), Frecuentemente (4) y Siempre (5).

El proceso de validación de las escalas Likert se centró en la determinación de la validez del contenido, que busca asegurar que cada cuestionario mida lo que pretende medir. Para ello, una vez confeccionado el instrumento fue entregado a un grupo de 3 expertos, especializados en el área de la metodo-logía y didáctica de la matemática en la educación superior. A cada experto se le facilitó la información relacionada con los objetivos, planteamiento del problema y el sistema de variables de la investigación, así como un formato para poder plasmar sus criterios. Cada experto hizo sugerencias en relación con los criterios sugeridos por Ruíz (2002), que hacen referencia a la claridad, congruencia y tendenciosidad de cada uno de los ítemes que conformaron las escalas del cuestionario. Estas sugerencias fueron discutidas y adoptadas hasta que se logró conformar la versión definitiva del cuestionario que se empleó en la recolección de la información.

Dada la naturaleza de las escalas para calcular el índice de confiabilidad, se realizó una prueba piloto con veinte participantes para determinar el índice de confiabilidad Alfa de Cronbach; lo que arrojó un valor de 0,90; por lo que se considera, de acuerdo con Ruíz Bolívar (1999), un instrumento confiable que avalan su utilización.

\section{Resultados y discusiones}

Análisis descriptivo de las estrategias referidas a la planificación, desarrollo y evaluación del proceso de enseñanza: se presentan los resultados de la opinión de los estudiantes con respecto a la frecuencia con la que los docentes emplean las estrategias del proceso de enseñanza de la matemática.
La tabla 1 muestra la distribución de los estudiantes que participaron en el estudio, de acuerdo con su conocimiento acerca de las estrategias de enseñanza. El 70 \% del grupo manifiesta tener conocimiento sobre estrategias de enseñanza.

Tabla 1. Distribución del grupo de estudiantes sobre experiencia en estrategias de enseñanza

\begin{tabular}{ccccccc}
\hline & \multicolumn{2}{c}{ No } & \multicolumn{2}{c}{ Sí } & \multicolumn{2}{c}{ Total } \\
& f & $\%$ & f & \% & f & \% \\
\hline $\begin{array}{c}\text { Posee algún tipo de } \\
\text { conocimiento sobre } \\
\text { estrategias de enseñanza }\end{array}$ & $\mathbf{1 5}$ & $\mathbf{2 9 , 4}$ & $\mathbf{3 6}$ & $\mathbf{7 0 , 6}$ & $\mathbf{5 1}$ & $\mathbf{1 0 0}$ \\
\hline
\end{tabular}

En las tablas 2, 3 y 4 se muestra la opinión de los estudiantes con respecto a la planificación, el desarrollo de estrategias y los aspectos evaluativos que perciben de sus docentes, durante el transcurso de las clases.

Tabla 2. Planificación de actividades para desarrollar en el aula

\begin{tabular}{|c|c|c|c|c|c|c|}
\hline & & Nunca & $\begin{array}{c}\text { Rara } \\
\text { vez }\end{array}$ & $\begin{array}{c}\begin{array}{c}\text { Algunas } \\
\text { veces }\end{array} \\
\end{array}$ & $\begin{array}{l}\text { Frecuen } \\
\text { te mente }\end{array}$ & Siempre \\
\hline \multirow{2}{*}{$\begin{array}{l}\text { 4. Tiene en cuenta las dificultades que } \\
\text { presentan los distintos contenidos } \\
\text { matemáticos a desarrollar. }\end{array}$} & f & 2 & 2 & 14 & 22 & 11 \\
\hline & $\%$ & $3,90 \%$ & $3,90 \%$ & $27,50 \%$ & $43,10 \%$ & $21,60 \%$ \\
\hline \multirow{2}{*}{$\begin{array}{l}\text { 5. Se informa de las trayectorias de los } \\
\text { estudiantes, a lo largo de los semestres } \\
\text { académicos }\end{array}$} & f & 4 & 7 & 14 & 18 & 8 \\
\hline & $\%$ & $7,80 \%$ & $13,70 \%$ & $27,50 \%$ & $35,30 \%$ & $15,70 \%$ \\
\hline \multirow{2}{*}{$\begin{array}{l}\text { 6. Tiene en cuenta carencias cognitivas } \\
\text { comunes, que presentan los estudiantes en } \\
\text { este nivel. }\end{array}$} & $\mathrm{f}$ & 3 & 6 & 16 & 21 & 5 \\
\hline & $\%$ & $5,90 \%$ & $11,80 \%$ & $31,40 \%$ & $41,20 \%$ & $9,80 \%$ \\
\hline \multirow{2}{*}{$\begin{array}{l}\text { 7. Considera el currículo vigente y lo integra } \\
\text { en la planificación de las actividades y en la } \\
\text { formulación de las competencias a } \\
\text { desarrollar. }\end{array}$} & $\mathrm{f}$ & 2 & 3 & 12 & 17 & 17 \\
\hline & $\%$ & $3,90 \%$ & $5,90 \%$ & $23,50 \%$ & $33,30 \%$ & $33,30 \%$ \\
\hline \multirow{2}{*}{$\begin{array}{l}\text { 8. Considera el perfil de personalidad y } \\
\text { profesional del grupo. }\end{array}$} & f & 2 & 1 & 11 & 18 & 19 \\
\hline & $\%$ & $3,90 \%$ & $2,00 \%$ & $21,60 \%$ & $35,30 \%$ & $37,30 \%$ \\
\hline \multirow{2}{*}{$\begin{array}{l}\text { 9. Considera los conocimientos previos de los } \\
\text { estudiantes. }\end{array}$} & f & 2 & 1 & 9 & 19 & 20 \\
\hline & $\%$ & $3,90 \%$ & $2,00 \%$ & $17,60 \%$ & $37,30 \%$ & $39,20 \%$ \\
\hline \multirow{2}{*}{$\begin{array}{l}\text { 10. Tiene en cuenta los recursos necesarios } \\
\text { para el desarrollo de cada tema. }\end{array}$} & f & 1 & 4 & 9 & 27 & 10 \\
\hline & $\%$ & $2,00 \%$ & $7,80 \%$ & $17,60 \%$ & $52,90 \%$ & $19,60 \%$ \\
\hline \multirow{2}{*}{$\begin{array}{l}\text { 11. Tiene en cuenta el tiempo que debo } \\
\text { dedicar a cada tarea o actividad. }\end{array}$} & f & 2 & 4 & 8 & 19 & 18 \\
\hline & $\%$ & $3,90 \%$ & $7,80 \%$ & $15,70 \%$ & $37,30 \%$ & $35,30 \%$ \\
\hline \multirow{2}{*}{$\begin{array}{l}\text { 12. Tiene en cuenta los microcurrículos } \\
\text { establecidos por los departamentos. }\end{array}$} & f & 3 & 1 & 7 & 25 & 15 \\
\hline & $\%$ & $5,90 \%$ & $2,00 \%$ & $13,70 \%$ & $49,00 \%$ & $29,40 \%$ \\
\hline \multirow{2}{*}{$\begin{array}{l}\text { 13. Promueve la interacción y participación } \\
\text { de los estudiantes. }\end{array}$} & $\mathrm{f}$ & 1 & 1 & 4 & 19 & 26 \\
\hline & $\%$ & $2,00 \%$ & $2,00 \%$ & $7,80 \%$ & $37,30 \%$ & $51,00 \%$ \\
\hline
\end{tabular}

La tabla 2 muestra los ítemes relacionados con la percepción que tienen los estudiantes en referencia a las actividades de planificación de la práctica docente en el aula. En general, destaca una presencia significativa en todos los indicadores analizados. Resaltan como indicadores evidenciados con un nivel alto, el hecho de que frecuentemente (87\%) los estudiantes perciben que sus docentes promueven la interacción y la participación en el aula; asímismo, consideran que toman en cuenta los microcurrículos establecidos por el departamento académico al que pertenecen (78 \%). En este sentido, aproximadamente el $72 \%$ de los estudiantes aprecian que, con mucha frecuencia, los docentes toman en cuenta el tiempo que deben 
dedicar a cada actividad, así como los recursos necesarios para el desarrollo de las mismas.

Por otra parte, llama la atención que el $28 \%$ de los estudiantes consideran que, solo algunas veces, los docentes toman en cuenta la dificultad de los contenidos que se desarrollarán y no toman en cuenta la trayectoria de los estudiantes en semestres anteriores. Aproximadamente una quinta parte del grupo considera que los docentes toman en cuenta aspectos relacionados con el currículo o los perfiles de personalidad y profesional del grupo. Aproximadamente el $21 \%$ del grupo encuentra que rara vez se toman en consideración aspectos individuales de los estudiantes o sus carencias cognitivas (18\%).

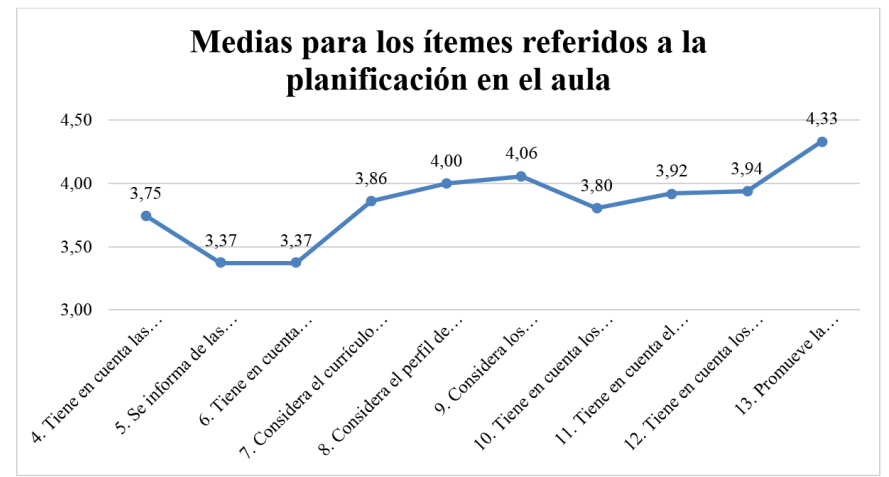

Figura 1. Medias para los ítemes referidos a la planificación en el aula

La figura 1 evidencia que las medias más altas son las relacionadas con la interacción $(4,33)$ y la planificación del tiempo y de los recursos (3,80 y 3,92 respectivamente). Los aspectos relacionados con la consideración de los aspectos individuales de cada estudiante, son los que tienen medias más bajas $(3,37)$.

De acuerdo a lo anterior es recomedable que los docentes conozcan los estilos de aprendizaje que tienen sus estudiantes, ya que estos son de vital importancia para poder potenciar su proceso de enseñanza- aprendizaje (Reza, Calderón, Miranda, $\&$ Gallegos, 2018). Por ello es necesario tomarlos en cuenta al momento de planificar, ejecutar y evaluar las clases (Castro \& Guzmán, 2005).

Tabla 3. Desarrollo de estrategias al momento de ejecutar las actividades de aula

\begin{tabular}{|c|c|c|c|c|c|c|}
\hline & & Nunca & Rara vez & $\begin{array}{c}\text { Algunas } \\
\text { veces }\end{array}$ & $\begin{array}{c}\text { Frecuent } \\
\text { emente }\end{array}$ & Siempre \\
\hline \multirow{2}{*}{$\begin{array}{c}\text { 14. Facilita a los estudiantes las } \\
\text { orientaciones necesarias, para dar inicio } \\
\text { a una nueva tarea o actividad de } \\
\text { aprendizaje }\end{array}$} & $\mathrm{f}$ & 2 & 1 & 6 & 25 & 17 \\
\hline & $\%$ & $3,90 \%$ & $2,00 \%$ & $11,80 \%$ & $49,00 \%$ & $33,30 \%$ \\
\hline \multirow{2}{*}{$\begin{array}{l}\text { 15. Utiliza ejemplos, no ejemplos y } \\
\text { contraejemplos para crear una imagen } \\
\text { de los conceptos matemáticos }\end{array}$} & $\mathrm{f}$ & 1 & 2 & 11 & 14 & 23 \\
\hline & $\%$ & $2,00 \%$ & $3,90 \%$ & $21,60 \%$ & $27,50 \%$ & $45,10 \%$ \\
\hline \multirow{2}{*}{$\begin{array}{l}\text { 16. Promueve actividades matemáticas } \\
\text { centradas en la resolución de problemas. }\end{array}$} & $\mathrm{f}$ & 3 & 3 & 11 & 20 & 14 \\
\hline & $\%$ & $5,90 \%$ & $5,90 \%$ & $21,60 \%$ & $39,20 \%$ & $27,50 \%$ \\
\hline \multirow{2}{*}{$\begin{array}{l}\text { 17. Promueve actividades matemáticas } \\
\text { centradas en el establecimiento de } \\
\text { conjeturas }\end{array}$} & $\mathrm{f}$ & 2 & 5 & 17 & 22 & 5 \\
\hline & $\%$ & $3,90 \%$ & $9,80 \%$ & $33,30 \%$ & $43,10 \%$ & $9,80 \%$ \\
\hline \multirow{2}{*}{$\begin{array}{l}\text { 18. Promueve actividades matemáticas } \\
\text { centradas en la demostración. }\end{array}$} & f & 2 & 3 & 8 & 18 & 20 \\
\hline & $\%$ & $3,90 \%$ & $5,90 \%$ & $15,70 \%$ & $35,30 \%$ & $39,20 \%$ \\
\hline \multirow{2}{*}{$\begin{array}{l}\text { 19. Promueve actividades matemáticas } \\
\text { centradas en la formalización de } \\
\text { conceptos. }\end{array}$} & f & 2 & 0 & 10 & 24 & 15 \\
\hline & $\%$ & $3,90 \%$ & $0,00 \%$ & $19,60 \%$ & $47,10 \%$ & $29,40 \%$ \\
\hline
\end{tabular}

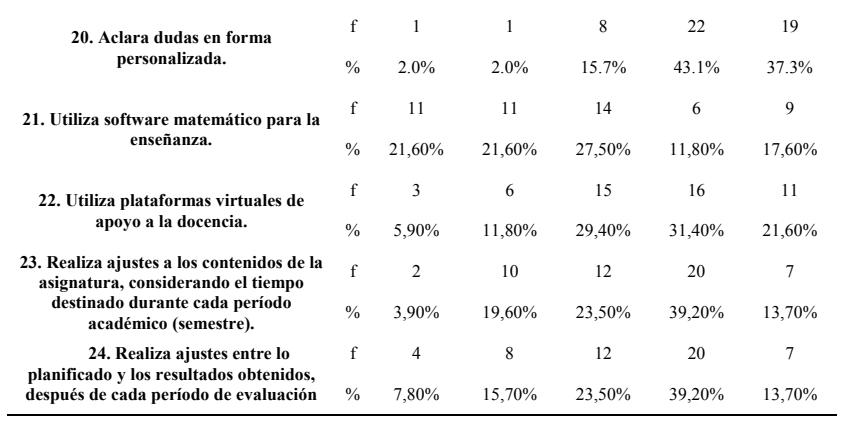

La tabla 3 relaciona los ítemes referidos al desarrollo de estrategias que se ejecutan en el aula. Entre de los indicadores destacados por los estudiantes, sobresale el hecho de que sus docentes en forma frecuente, aclaran dudas en forma personalizada $(80 \%)$ y que promueven la realización de actividades matemáticas centradas en la formalización de conceptos (76 $\%$ ). También perciben en una proporción elevada la promoción de actividades basadas en la demostración (74\%) y el uso de ejemplos y contraejemplos (72\%).

Un tercio de los estudiantes está de acuerdo en afirmar que, sólo algunas veces se promueven actividades centradas en el establecimiento de conjeturas; asimismo un 29,4\% reporta que solo algunas veces se utilizan plataformas virtuales de apoyo a la docencia. Por otra parte, los estudiantes reportaron que ocasionalmente, incluso nunca, se utiliza software matemático para la enseñanza (43\%) y que se realizan ajustes a los contenidos o a la planificación de acuerdo con los resultados de las evaluaciones $(23 \%)$.

\section{Medias para los ítemes referidos al desarrollo de estrategias}

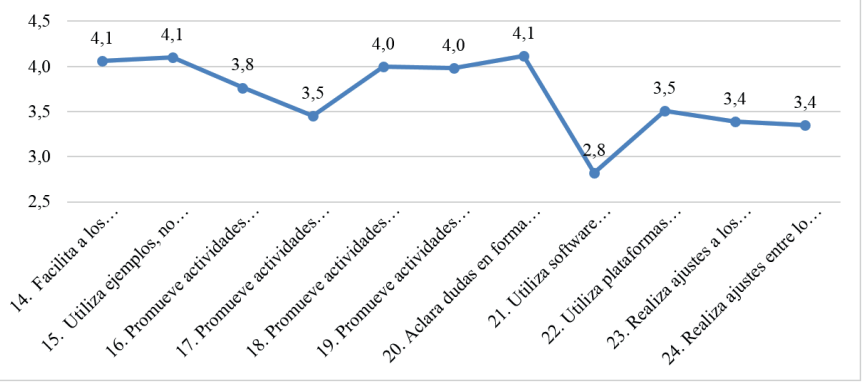

Figura 2. Medias para los ítemes referidos al desarrollo de estrategias

En la figura 2 se muestran las medias aritméticas para las escalas, en función de las respuestas en cada indicador. Se puede observar que el valor más bajo $(2,82)$ está relacionado con el uso de software matemático para la enseñanza, mientras que las estrategias basadas en la interacción directa con el estudiante para aclarar las dudas $(4,12)$ y el uso de ejemplos $(4,10)$ son las más valoradas por el grupo de alumnos evaluados.

De acuerdo a lo establecido es necesario potenciar en la practica pedagógica el uso de las TIC, entre ellas el software matemático o las aplicaciones matemáticas, ya que los docentes y estudiantes encuentran en ellas, herramientas que permi- 
ten mejorar los procesos de enseñanza y aprendizaje de forma eficaz en esta área del conocimiento (Mosquera \& Vivas, 2017). Ademas, el software educativo puede ser caracterizado no solo como recurso de enseñanza y aprendizaje sino también como una estrategia de enseñanza; así el uso de un determinado software conlleva unas estrategias de aplicación implícitas o explicitas: ejercitación y práctica, simulación, tutorial, uso individual, competición, pequeño grupo (Fernández, Riveros, \& Montiel, 2017).

De igual manera, se debe aumentar el uso de la plataforma de apoyo a la docencia por parte de los profesores ya que esto permite impulsar el desarrollo de la innovación educativa mediante el uso de las TIC y producir una transformación de las aulas virtuales, para dejar de ser solamente repositorios de información, y dar lugar a una docencia virtual activa, participativa y colaborativa (Fariña-Vargas, González-González, \& Area-Moreira, 2013; Prada, Hernández \& Gamboa, 2019); por eso es necesario definir las estrategias de la educación virtual de acuerdo al programa academico y las microcurriculos que se desean implementar (Barrera \& Guapi, 2018), en este caso, el de formación inicial de docentes de matemáticas.

Tabla 3. La evaluación de los aprendizajes en el aula

\begin{tabular}{|c|c|c|c|c|c|c|}
\hline & & Nunca & $\begin{array}{c}\text { Rara } \\
\text { vez }\end{array}$ & $\begin{array}{l}\text { Algunas } \\
\text { veces }\end{array}$ & Frecuentemente & Siempre \\
\hline \multirow{2}{*}{$\begin{array}{l}\text { 25. Practica la evaluación formativa, } \\
\text { coevaluación y autoevaluación en los } \\
\text { estudiantes. }\end{array}$} & f & 22 & 3 & 3 & 12 & 11 \\
\hline & $\%$ & $43,10 \%$ & $5,90 \%$ & $5,90 \%$ & $23,50 \%$ & $21,60 \%$ \\
\hline \multirow{2}{*}{$\begin{array}{l}\text { 26. Practica la coevaluación en los } \\
\text { estudiantes. }\end{array}$} & f & 21 & 4 & 10 & 10 & 6 \\
\hline & $\%$ & $41,20 \%$ & $7,80 \%$ & $19,60 \%$ & $19,60 \%$ & $11,80 \%$ \\
\hline \multirow{2}{*}{$\begin{array}{l}\text { 27. Practica la autoevaluación en los } \\
\text { estudiantes. }\end{array}$} & f & 14 & 11 & 8 & 7 & 11 \\
\hline & $\%$ & $27,50 \%$ & $21,60 \%$ & $15,70 \%$ & $13,70 \%$ & $21,60 \%$ \\
\hline \multirow{2}{*}{$\begin{array}{l}\text { 28. Utilizo los resultados de la } \\
\text { evaluación del aprendizaje, para } \\
\text { reflexionar sobre mi trabajo. }\end{array}$} & $\mathrm{f}$ & 18 & 4 & 6 & 7 & 16 \\
\hline & $\%$ & $35,30 \%$ & $7,80 \%$ & $11,80 \%$ & $13,70 \%$ & $31,40 \%$ \\
\hline \multirow{2}{*}{$\begin{array}{l}\text { 29. Utiliza los resultados de la } \\
\text { evaluación para hacer } \\
\text { modificaciones en el proceso de } \\
\text { enseñanza. }\end{array}$} & $f$ & 17 & 4 & 8 & 8 & 14 \\
\hline & $\%$ & $33,30 \%$ & $7,80 \%$ & $15,70 \%$ & $15,70 \%$ & $27,50 \%$ \\
\hline \multirow{2}{*}{$\begin{array}{l}\text { 30. Comunica oportunamente los } \\
\text { resultados del proceso de evaluación. }\end{array}$} & $\mathrm{f}$ & 12 & 2 & 3 & 4 & 30 \\
\hline & $\%$ & $23,50 \%$ & $3,90 \%$ & $5,90 \%$ & $7,80 \%$ & $58,80 \%$ \\
\hline \multirow{2}{*}{$\begin{array}{l}\text { 31. Comunica los resultados de las } \\
\text { pruebas escritas, señalando los } \\
\text { errores y realizando las correcciones } \\
\text { que tuvieran lugar. }\end{array}$} & $\mathrm{f}$ & 12 & 2 & 4 & 11 & 22 \\
\hline & $\%$ & $23,50 \%$ & $3,90 \%$ & $7,80 \%$ & $21,60 \%$ & $43,10 \%$ \\
\hline \multirow{2}{*}{$\begin{array}{l}\text { 32. Propone una colección de } \\
\text { tareas comunes para la nivelación de } \\
\text { los estudiantes que no han alcanzado } \\
\text { los objetivos previstos. }\end{array}$} & $\mathrm{f}$ & 16 & 3 & 7 & 11 & 14 \\
\hline & $\%$ & $31,40 \%$ & $5,90 \%$ & $13,70 \%$ & $21,60 \%$ & $27,50 \%$ \\
\hline \multirow{2}{*}{$\begin{array}{l}\text { 33. Establece un plan de trabajo } \\
\text { individualizado para los estudiantes } \\
\text { que no han alcanzado los objetivos } \\
\text { previstos y realiza el seguimiento. }\end{array}$} & $\mathrm{f}$ & 9 & 10 & 7 & 14 & 11 \\
\hline & $\%$ & $17,60 \%$ & $19,60 \%$ & $13,70 \%$ & $27,50 \%$ & $21,60 \%$ \\
\hline
\end{tabular}

Los ítemes relacionados con la evaluación de los aprendizajes en el aula, se presentan en la tabla 3 . En general se observa una distribución equitativa de opiniones variadas que van desde nunca hasta siempre. Por una parte, destaca con aproximadamente el $65 \%$ de las opiniones, el hecho de que frecuentemente los estudiantes consideran que les comunican oportunamente el resultado de las evaluaciones, señalando los errores y correcciones respectivas. Sin embargo, menos de la mitad del grupo $(48 \%)$ considera que, frecuentemente se proponen actividades de nivelación o un plan de trabajo individualizado y se le hace seguimiento.

Aproximadamente un $70 \%$ del grupo cuestiona la forma en que se lleva a cabo la coevaluación en el aula y un $49 \%$ opina que rara vez observa actividades de evaluación formativa y autoevaluación, así como se cuestiona el uso de los resultados para reflexionar sobre el trabajo en el aula (43\%). Destaca por otra parte, que un $37 \%$ del grupo indica que nunca o en raras ocasiones se proponen actividades de nivelación.

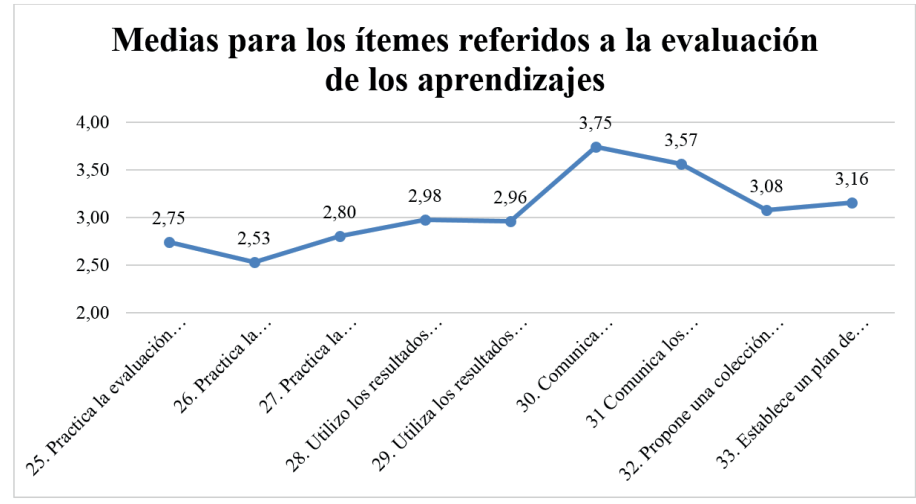

Figura 3. Medias para los ítemes referidos a la evaluación de los aprendizajes

La figura 3 permite contrastar las apreciaciones descriptivas y se observa que los indicadores con medias más altas $(3,75$ y 3,57$)$ son los relacionados con la comunicación de los resultados, mientras que los de medias más bajas $(2,75$ y 2,53$)$ se asocian a la implementación de la coevaluación y autoevaluación en el aula.

Por lo anterior, se hace necesario que la evaluación sea integral y participativa, centrada en la utilidad de los aprendizajes más que en el contenido, donde los estudiantes evalúen los procesos de su formación y tomen conciencia de los logros alcanzados (Fernandez \& Vanga, 2015) por lo que se debe potenciar la coevaluación, como una alternativa complementaria para evaluar determinadas competencias de los estudiantes universitarios (Gessa, 2011) y la autoevaluación por parte del estudiante, como un proceso que puede ser más efectivo, eficiente y pertinente para medir la asimilación de conocimientos guiado y monitoreado permanentemente por el docente (Camacho, 2014).

\section{Conclusiones}

A nivel global, la valoración de las estrategias de enseñanza aplicadas por los docentes a cargo de asignaturas de matemáticas por parte de los estudiantes resultó ser positiva.

Sin embargo, se manifiesta que los docentes no consideran las necesidades individuales y las trayectorias académicas de los estudiantes. Es decir, los docentes centran su planificación en el desarrollo de los contenidos sin considerar las necesidades de los estudiantes. 
Por otra parte, aun cuando los estudiantes destacan que, a pesar de recibir apoyo personalizado fuera del aula, se encuentran desasistidos, pues casi no se promueve el uso de los espacios virtuales con los que se cuenta. Ademas, del poco uso que se hace del software educativo, especialmente en matemáticas, el cual debería ser un elemento fundamental en la formación del docente.

Finalmente, en el caso de la evaluación, pareciera que esta solo se constituye en la aplicación de pruebas de carácter sumativo; que no se utiliza como fuente para proyectar mejoras, dejando de ser integral, participativa y transformadora que valore el desempeño estudiantil durante su formación profesional.

\section{Referencias}

Barrera, V., \& Guapi, A. (2018 ). La importancia del uso de las plataformas virtuales en la educación superior.

Atlante Cuadernos de Educación y Desarrollo.

Blanco, L. (2011). La investigación en Educación Matemática. Educatio Siglo XXI, 29(1), 109-128.

Blum, W. (1985). Awendungsorientierter Mathematikunterricht in der didaktischen Diskussion. Mathematische Se-

mesterberichte(32), 195-232.

Bretones, A. (1996 ). Concepciones y practicas de participación en el aula segun los estudiantes de magisterio (tesis doctoral). Madrid, España: Universidad Complutense.

Camacho, C. (2014). La autoevaluación en el aula: Un método innovador para mejorar la calidad del proceso enseñanza-aprendizaje en la formación de maestras y maestros. Revista Integra Educativa, 7(2), 159-172.

Castro, S., \& Guzmán , B. (2005). Los estilos de aprendizaje en la enseñanza y el aprendizaje: Una propuesta para su implementación. Revista de Investigación(58), 83-102.

Covarrubias , P., \& Piña, M. (2004). La interacción maestro-alumno y su relación con el aprendizaje. Revista Latinoamericana de Estudios Educativos, 34(1), 47-84.

Do Ponte, J., Brunheira, L., Abrantes, P., \& Bastos, R. (1998). Matemática. Projectos Educativos. Lisboa: Ministério do Educação.

Fariña-Vargas, E., González-González, C., \& Area-Moreira, M. (2013). ¿Qué uso hacen de las aulas virtuales los docentes universitarios? Revista de Educación a Distancia $R E D(35), 1-13$.

Feo, R. (2010). Orientafciones básicas para el diseño de estrategias didácticas. Tendencias pedagógicas(16), 220-236.

Fernandez, A., \& Vanga, M. (2015). Proceso de autoevaluación, coevaluación y heteroevaluación para caracterizar el comportamiento estudiantil y mejorar su desempeño. Revista San Gregorio, 1(9), 6-15.

Fernández, I., Riveros, V., \& Montiel., G. (2017). Software educativo y las funciones matemáticas. Una estrategia de apropiación de sus procesos. Omnia, 23(1), 9-19. mentaria de la evaluación del aprendizaje: análisis y reflexión en las aulas universitarias. Revista de educación, 354, 749-764.

Jiménez, A. (2007 ). Didáctica y educación matemática. En Rojas, P. Memorias del $8^{\circ}$ Encuentro Colombiano de Matemática Educativa (págs. 15-22). Cali: Gaia.

Marchesi, A., Tedesco, J., \& Coll, C. (2009). Calidad, equidad y reformas en la enseñanza. Madrid, España: OEI - Fundación Santillana.

Mora, C. (2003). Estrategias para el aprendizaje y la enseñanza de las matemáticas. Revista de pedagogía, 24(70), 181272.

Mosquera, M., \& Vivas, S. (2017). Análisis comparativo de software matemático para la formación de competencias de aprendizaje en cálculo diferencial. Plumilla Educativa, 19(1), 98-113.

Muñiz-Rodríguez, L., Alonso, P., \& Rodríguez-Muñiz , L. (2014). El uso de los juegos como recurso didáctico para la enseñanza y el aprendizaje de las Matemáticas: estudio de una experiencia innovadora. Revista Iberoamericana de Educación Matemática(39), 19-33.

Pardo, A., \& Ruiz, M. (2002). SPSS 11: guía para el análisis de datos. Madrid: Mc Graw Hill.

Prada, R., Hernández, C. \& Gamboa , A. (2019 ). Usos y efectos de la implementación de una plataforma digital en el proceso de enseñanza de futuros docentes en matemáticas. Revista Virtual Católica del Norte , 57(2), 137-156.

Reza, L., Calderón, P., Miranda, R., \& Gallegos, Y. (2018). Estilos de aprendizaje de los estudiantes de la Unidad Educativa Fiscal "Dolores Cacuango" y su influencia en el rendimiento académico. Revista Atlante. Cuadernos de Educación y Desarrollo.

Ruíz, C. (2002). Instrumentos de Investigacion Educativa: Procedimientos para su Diseño y Validación. Barquisimeto, Venezuela: Ediciones CIDEG.

Sánchez, J., \& Fernández, J. (2003). La enseñanza de la matemática : fundamentos teóricos y bases psicopedagógicas. Madrid: Editorial CCS.

Serres, Y. (2002). La demostración en educación matemática. Mimeografiado. Caracas: Universidad Central de Venezuela.

Gessa, A. (2011). La coevaluación como metodología comple-

$53 \quad$ Perspectivas, 4 (1), pp. 48-53, 2019, ISSN 2590-9215 